\title{
PRODUÇÃO E AVALIAÇÃO DE ATIVIDADE BIOLÓGICA DE POMADA A BASE DE EXTRATO DE Anadenanthera colubrina (Vell.) Brenan
}

\author{
Beatriz Santana Rocha ${ }^{1 *}$, Maria Giuliane Gonçalves da Sila ${ }^{1}$, Rafael Jorge Santos Aracati Padilha ${ }^{1}$, Elizabete Regina \\ Silva Lucena dos Santos ${ }^{1}$, Leylianne de Cassia Rodrigues Nerys ${ }^{1}$, Kêsia Xisto da Fonseca Ribeiro de Sena ${ }^{2}$, Cláudia \\ Sampaio de Andrade Lima ${ }^{2}$, Ricardo Yara ${ }^{3}$
}

${ }^{1}$ Laboratório de Biofísica Química, UFPE; 'Laboratório de Antibióticos, UFPE; ${ }^{3}$ Laboratório de Engenharia Biomédica, UFPE *beatrizsantanarocha@gmail.com

\section{INTRODUÇ̃̃O}

A comercialização de fitoterápicos e plantas medicinais vem se destacando no mercado nacional, pois fortalece a produção local com base no manejo da rica biodiversidade (HASENCIEVER et al., 2017). Dentre os biomas encontrados no país, a caatinga é o menos estudado, contudo, fora identificado grande variedade bioló gica na região (MOURA et al, 2016). Abrange desde atividades econômicas voltadas para fins agrosilvopastoris, até industriais, destacando-se as áreas farmacêuticas, químicas, alimentícias e de cosméticos (MMA, 2017).

Dentre a vasta coleção biológica da caatinga, destaca-se a espécie Anadenanthera colubrina (Vellozo) Brenan, pertencente à subfamília Mimosoideae (Leguminosae), popularmente conhecida como angico. É uma árvore comumente utilizada na arborização de ambientes, mas também muito empregada na confecção de tacos, ripas, lenha e carvão de boa qualidade (GARCIA, 2004). Em relação ao seu potencial terapêutico, já é utilizada como aliada no tratamento de cérvico-vaginites, blenorragias, tosses e bronquites (MONTEIRO et al., 2006 \& MORS et al., 2000).

A fitocosmética é o segmento da ciência cosmetológica que se dedica a pesquisa e aplicação dos conhecimentos da ação dos princípios ativos extraídos de vegetais, com objetivos desde higiene e estético, até de manter a homeostasia de tecidos (ARAÚ JO et al., 2010). Algumas formulações, como as pomadas, destacam por terem maior capacidade de transferir componentes com potencial terapêutico ao local em que foi aplicado, quando comparado a outras formulações que contenham a mesma quantidade destes (PERSHING, 1992).

Assim, com base no potencial terapêutico já encontrado no angico, aliado aos princípios da fitocosmética, o objetivo deste trabalho foi avaliar a atividade antimicrobiana do extrato e atividade antioxidade da união entre uma nova formulação de pomada e o extrato etanólico de Anadenanthera colubrina (Vell.) Brenan.

\section{MATERIAIS E MÉTODOS}

As cascas da espécie Anadenanthera colubrina (Vell.) Brenan foram coletadas no município de Altinho, no estado de Pernambuco. Em seguida, o material foi acondicionado em estufa de secagem, expostas a uma temperatura de $42^{\circ} \mathrm{C}$ com circulação forçada de ar. Após trituração e filtração, o material foi destilado a vácuo em evaporador rotativo (RV 10 Control da IKA), com temperatura máxima de $45^{\circ} \mathrm{C}$ para a obtenção do extrato bruto que, posteriormente, foi armazenado em dessecador para que fosse atingido peso constante. Já para confecção das pomadas foram utilizados $1,25 \mathrm{~g}$ de vaselina, $1,25 \mathrm{~g}$ de lanolina e variadas concentrações do extrato separadamente: 0,25g (Pomada 10\%), 0,125g (Pomada 5\%) e 0,025g (Pomada 1\%)

Para análise da atividade antioxidante foi realizado o teste de redução do DPPH (2,2-difenil-1- picrilhidrazil). Este baseia-se na transferência de elétrons onde, por ação antioxidante, altera a cor da solução de púrpura para amarelo. Apó $\mathrm{s}$ a adição do DPPH nas soluções, esperou-se 30 minutos, ao abrigo da luz, para a realização da leitura em espectrofotômetro de UVVis a $515 \mathrm{~nm}$. A capacidade de eliminar o radical DPPH (\% de atividade antioxidante) foi calculada utilizando-se a seguinte equaç ão:

Eliminação $[\mathrm{DPPH}](\%)=\underline{(\text { Abs amostra }- \text { Abs controle })} \times 100$ Abs controle

Onde: Abs (Absorbância)

A partir dos resultados obtidos determinou-se a porcentagem de atividade antioxidante ou sequestradora de radicais livres (NASCIMENTO et al., 2011).

0 teste foi feito separadamente com a lanolina, a vaselina, o extrato etanólico e a pomada, todas na concentração de $1000 \mu \mathrm{g} / \mathrm{ml}$. Um controle negativo foi feito pela adição de etanol e DPPH. Adicionou-se a cada amostra analisada uma solução de DPPH a $12 \%$, exceto nos brancos, onde foi utilizado apenas o solvente (NASCIMENTO et al., 2011). Os testes foram feitos em triplicata.

Já os testes para a determinação da atividade antimicrobiana foram realizados de acordo com o método de difusã o em disco (BAUER et al., 1966), com modificações, no laboratório do Departamento de Antibióticos da UFPE (UFPEDA), com os seguintes micro-organismos: Staphylococcus aureus (UFPEDA 01), Mycrococcus luteus (UFPEDA 06), Bacillus subtilis (UFPEDA 16), Pseudomonas aeruginosa (UFPEDA 39), Mycobacterium smegmatis (UFPEDA 71), Enterococcus faecalis (UFPEDA 138), Escherichia coli (UFPEDA 224), e a levedura Candida albicans (UFPEDA 1007).

Foram utilizados $200 \mathrm{mg}$ do extrato, para a diluição foi utilizada uma solução de $2 \mathrm{mg} / \mathrm{ml}$ de DMSO, e distribuídos em discos de papel de filtro esterilizados de $6 \mathrm{~mm}$ de diâmetro. Os discos foram postos sobre o meio de cultura correspondente para cada micro-organismo, a suspensão do inóculo dos microorganismos em solução salina correspondeu a 0,5 na escala de 
McFarland, sendo os micro-organismos semeados em meio ágar Muller-Hinton (g/L): extrato de carne 30; caseína hidrolisada 17,5; amido 1,5; agar 17 e $\mathrm{pH}$ final $7,3 \mathrm{e} \mathrm{GL}(\mathrm{g} / \mathrm{L})$ : peptona 10 ; extrato de carne 3; $\mathrm{NaCl} 5$; extrato de levedura 10; glicose ou dextrose 10; agar 15 e pH final de 7,0com um swab estéril, após, as placas foram colocadas em estufa a $45^{\circ} \mathrm{C}$ num período de 24 horas, e apó $\mathrm{s}$, foi realizada a observação e medição dos halos. Os controles positivos utilizados foram: Ciclofosfamida (CTX), Cefoxitina (CFO) e Clorafenicol (CLO). O controle negativo foram placas com apenas os microorganismos. Os testes foram realizados em triplicata.

\section{RESULTADOS E DISCUSSÃO}

A escolha dos excipientes utilizados na composição das pomadas justifica-se pelo fato da lanolina apresentar caracterí sticas emolientes e por ser doador de consistência, e da vaselina permitir a lubricidade na aplicação sobre a pele. A união destas confere maior eficácia na absorção dos princípios ativos (CÂNDIDA, 2003). Além disso, esta a associação resulta numa mistura hidrofó bica, a qual causa efeito oclusivo (vaselina) e ação endodérmica (lanolina), com penetração cutânea, o que melhora a ação em camadas mais profundas, sem afetar a corrente circulatória (BORELLA, 2010).

A análise dos resultados comparou a atividade antioxidante entre o extrato bruto da casca do angico, a lanolina, a vaselina e as pomadas produzidas. Como controle positivo foi escolhido o ácido ascórbico por ser uma substância de grande atividade antioxidante, além de ser um dos mais utilizados na rotina laboratorial encontrado na literatura (SHARIAH et al., 2013). A comparação entre as matérias-primas foi feita para justificar todas as possíveis origens da atividade antioxidante obtida na pomada (Tabela 1).

Tabela 1: Resultados de teste de redução de DPPH.

\begin{tabular}{c|c}
\hline Amostras analisadas & $\begin{array}{c}\text { Resultados do teste feito na } \\
\text { concentração de } 1000 \mu \mathrm{g} / \mathrm{ml}\end{array}$ \\
\hline Ácido Ascórbico & $95,85 \%$ \\
\hline Extrato etanólico de angico & $91,89 \%$ \\
\hline Vaselina & - \\
\hline Lanolina & - \\
\hline Pomada (5\%) & $90,88 \%$ \\
\hline Pomada (2,5\%) & $69,93 \%$ \\
\hline Pomada (1\%) & $11,08 \%$
\end{tabular}

Legenda: (-) Valores próximos a zero.

Com a realização do teste de redução de DPPH nas pomadas em diferentes concentrações de extratos, foi possível observar que, mesmo em concentrações baixas (5\%), são encontrados valores semelhantes aos visualizados no padrão (ácido ascórbico). O extrato e a pomada (5\%) apresentaram valores acima de $90 \%$, enquanto que a vaselina e lanolina não demonstraram nenhuma atividade, desse modo, pode-se atribuir ao extrato a atividade antioxidante encontrada em todas as pomadas produzidas.

O extrato da casca do angico já apresentara atividade antimicrobiana para todos os micro-organismos testados com exceç ão da levedura Candida Albicans (Tabela 2).

As atividades encontradas neste trabalho, podem ser conferidas a metabólitos secundários já detectados no angico como taninos (MONTEIRO et al, 2006), saponinas, triterpenos, açúcares redutores, antocianidinas e flavonoides (PESSOA et al., 2012). Como por exemplo a Anadantosídeo, flavonóide isolado das cascas de angico (Piacente et al., 1999). Diante desses resultados seu potencial antimicrobiano e antioxidante pode ser atribuído a compostos fenólicos já encontrados na espécie. Tais substâncias sã o de extremo interesse da indústria e da medicina tradicional, por apresentarem atividades terapêuticas.
Tabela 2: Resultados da avaliação antimicrobiana

\begin{tabular}{c|c}
\hline Micro-organismos testados & $\begin{array}{c}\text { Diâmetro dos Halos de inibição } \\
(\mathbf{m m})\end{array}$ \\
\hline Staphylococcus aureus & 21 \\
\hline Mycrococcus luteus & 24 \\
\hline Bacillus subtilis & 20 \\
\hline Pseudomonas aeruginosa & 12 \\
\hline Pseudomonas aeruginosa & 17 \\
\hline Enterococcus faecalis & 14 \\
\hline Escherichia coli & 10 \\
\hline Candida albicans & 0 \\
\hline
\end{tabular}

\section{CONCLUSÕES}

Os resultados obtidos neste trabalho revelaram que o angico tem um potencial antimicrobiano e antioxidante excelentes com valores próximos aos padrões testados. Tal fato, aliado com as características já descritas na literatura, como cicatrizante, são qualidades dos cosméticos mais comercializados atualmente, uma vez que, auxiliam no tratamento de injúrias, além de prevenir o envelhecimento da pele. Desse modo, o angico pode ser considerado um potencial fitocosmético, necessitando de testes farmacoquímicos subseqüentes para sua possível aplicação.

Apoio:

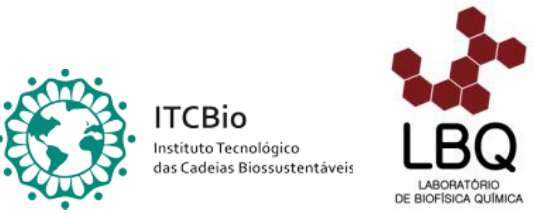

\section{REFERÊNCIAS}

ARAUJO, A.I.F. et. al. Plantas nativas do Brasil empregadas em fitocosmética. X Jornada de Ensino, Pesquisa e Extensão: 2010. BAUER AW, Kirby WM, TURCK M. Antibiotic susceptibility testing by a standardized single disk method. Amer J Clin Pathol. 1966; 45 (32): 493- 6.

BORELLA JC, RIBEIRO NS, TEIXEIRA JCL, CARVALHO DMA. Avaliação da espalhabilidade e do teor de flavonóides em forma farmacêutica semissólida contendo extratos de Calendyla officinalis $L$. (Asteraceae). Rev Ciênc Farm Básica Apl. 2010; 31 (2): 193-7

CÂNDIDA, M. Revista diversa, v.27, dezembro / janeiro 2003. Belo Horizonte, MG: Universitária, UFMG. 2003-. Anual. Disponível em: <www.ufmg.br/diversa.htm>.

GARCIA, L. C.; NOGUEIRA, A. C.; ABREU, D. C. A. Influência do envelhecimento acelerado no vigor de sementes de Anadenanthera colubrina (Vellozo) Brenan - Mimosaceae. Ciência Florestal, v.14, n.1, p.85-90, 2004

HASENCLEVER, LIA; PARANHOS, JULIA; REIS COSTA, CÍNTIA; CUNHA, GABRIEL; VIEIRA, DIEGO. A indústria de fitoterápicos brasileira: desafios e oportunidades Ciência \& Saúde Coletiva, vol. 22, núm. 8, agosto, 2017, pp. 2559-2569 Associação Brasileira de PósGraduação em Saúde Coletiva Rio de Janeiro, Brasil.

HEJMEN, F. H. et al. Cross-linking of dermal sheep collagen with tannic acid. Biomaterials, v. 18, n. 10, p. 749-754, 1997.

MMA - Ministério do Meio Ambiente. Caatinga. Disponível em: Acesso em: Nov 2017.

MONTEIRO, J.M.; ALMEIDA, C.F.R.; ALBUQUERQUE, U.P.; LUCENA, R.F.P. Use and tradicional management of Anadenanthera colubrina (Vell.) Brenan in the semi-arid region of northeastern Brazil. Journal of Ethnobiology and Ethnomedicine, v. 2, n. 6, p. 17, 2006.

MORS, W.B., RIZZINI, C.T., PEREIRA, N.A. Medicinal plants of Brazil. Michigan: Reference Publications, 2000. 
MOURA, M. M. S.; COSTA, G. B. R.; PALÁCIO, H. A. Q.; ARAÚJO NETO, J. R.; BRASIL, J. B. Produção de serapilheira e suas frações em área da Caatinga no Semiárido Tropical. Rev. Bras. Gest. Amb. Sustent. [online]. 2016, vol. 3, n. 5, p. 199-208. ISSN 2359-1412. DOI: $10.21438 /$ rbgas.030509.

NASCIMENTO J.C., LAGEL.F.O, CAMARGOS C.R.D, AMARAL J.C, COSTA L.M., SOUSA N.A, OLIVEIRA F.Q. Determinação da atividade antioxidante pelo método DPPH e doseamento de flavonóides totais em extratos de folhas da Bauhiniavariegata L. Rev. Bras. Farm.,v. 92 (4), P. 327-332, 2011.

PERSHING, L.K.; Silver, B.S.; Krueger, G.G.; Shah, V.P.; Skelley, J.P. Feasibility of measuring the bioavailability of topical betamethasone dipropionate in commercial formulations using drug content in skin and a skin blanching bioassay. Pharmaceutical Research, 1992.

PESSOA, Wagner Soares et al. Effects of angico extract (Anadenanthera colubrina var. cebil) in cutaneous wound healing in rats. Acta Cir. Bras., Oct 2012, vol.27, no.10, p.655-670. ISSN 0102-8650.

PIACENTE S, BALDERRAMA L, DE TOMMASI N ET AL. Anadanthoside: a flavanol-3-O-B-D-xylopyranoside from Anadenanthera macrocarpa. Phytochemistry, 51 (): 709, 1999.

SHARIAH, M. HOSSAI, M. I.; SHARMIM, F. A. AKHTER, S.; HAQUE, Md. A. BHUIYAN, M. A.In Vitro Antioxidant and free Radical Scavenging activity of WithaniaSommifera Root.losrJournal of Pharmacy, v.3 (2) p. 38-47, 2013. 